\title{
Towards a More Complete Understanding of SDC Propagation
}

\author{
Jon Calhoun \\ University of Illinois at Urbana-Champaign \\ 201 N. Goodwin Ave. \\ Urbana, Illinois 61801 \\ jccalho2@illinois.edu \\ Luke N. Olson \\ University of Illinois at Urbana-Champaign \\ 201 N. Goodwin Ave. \\ Urbana, Illinois 61801 \\ lukeo@illinois.edu
}

\author{
Marc Snir \\ University of Illinois at Urbana-Champaign \\ 201 N. Goodwin Ave. \\ Urbana, Illinois 61801 \\ snir@illinois.edu \\ William D. Gropp \\ University of Illinois at Urbana-Champaign \\ 201 N. Goodwin Ave. \\ Urbana, Illinois 61801 \\ wgropp@illinois.edu
}

\begin{abstract}
With the rate of errors that can silently effect an application's state/output expected to increase on future HPC machines, numerous application-level detection and recovery schemes have been proposed. Recovery is more efficient when errors are contained and affect only part of the computation's state. Containment is usually achieved by verifying all information leaking out of a statically defined containment domain, which is an expensive procedure. Alternatively, error propagation can be analyzed to bound the domain that is affected by a detected error. This paper investigates how silent data corruption (SDC) due to soft errors propagates through three HPC applications: HPCCG, Jacobi, and CoMD. To allow for more detailed view of error propagation, the paper tracks propagation at the instruction and application variable level. The impact of detection latency on error propagation is shown along with an application's ability to recover. Finally, the impact of compiler optimizations are explored along with the impact of local problem size on error propagation.
\end{abstract}

\section{KEYWORDS}

Silent Data Corruption; Error Propagation; Reliability; Error Detection; Error Recovery

\section{ACM Reference format:}

Jon Calhoun, Marc Snir, Luke N. Olson, and William D. Gropp. 2017. Towards a More Complete Understanding of SDC Propagation. In Proceedings of HPDC '17, Washington, DC, USA, June 26-30, 2017, 12 pages.

https://doi.org/http://dx.doi.org/10.1145/3078597.3078617

\section{INTRODUCTION}

Machine errors both hard and soft are expected to increase [42] as the number of components in processors increases and as chip

Permission to make digital or hard copies of all or part of this work for personal or classroom use is granted without fee provided that copies are not made or distributed for profit or commercial advantage and that copies bear this notice and the full citation on the first page. Copyrights for components of this work owned by others than ACM must be honored. Abstracting with credit is permitted. To copy otherwise, or republish, to post on servers or to redistribute to lists, requires prior specific permission and/or a fee. Request permissions from permissions@acm.org.

HPDC '17, fune 26-30, 2017, Washington, DC, USA

(C) 2017 Association for Computing Machinery.

ACM ISBN 978-1-4503-4699-3/17/06 \$ \$15.00

https://doi.org/http://dx.doi.org/10.1145/3078597.3078617 technologies, such as smaller feature sizes [9] or near-thresholdvoltage [26], are introduced to reduce power consumption. This places increased demands on both fabrication quality and on the underlying system and numerical software stacks.

Energetic particles from cosmic radiation can invert the state of transistors [3, 34]. Manufacturing defects can lead to the same effect. One consequence is that these faults produce soft errors that can cause a silent data corruption (SDC) - i.e., an erroneous deviation in system/application state. Corrupted state can be masked by the application causing no change in the output, or in extreme cases, can lead to corruption in the output. Modern hardware supports redundancy and techniques such as error correcting codes (ECC) to detect soft errors and to prevent them from affecting the computation state. As soft error rates increase, hardware may not provide adequate protection [43], allowing for corrupted state to impact application state. To combat this, software based SDC detection schemes have been developed, with many leveraging application properties and heuristics $[10,12,25]$. Others have taken application agnostic approaches $[5,6,8]$ or leverage various forms of redundancy [19, 28, 37].

Global checkpoint-restart is the de-facto fault tolerance protocol used by HPC applications to recover from fail-stop failures. The limited bandwidth of persistent storage is the main performance bottleneck of checkpointing methods. Current versions mitigate storage bandwidth issues by leveraging the memory hierarchy [ 7 , 35] and use compression techniques [40].

Another way to limit the impact of storage bandwidth is to avoid the need for global checkpointing and global restart. Checkpointrestart schemes often coordinate checkpointing and global restart: at checkpoint time, all processors are synchronized and the application state is saved, while at restart time, the entire application state is restored. This results in bursts of $\mathrm{I} / \mathrm{O}$ that slow down checkpoint and recovery. Various schemes have been proposed to support uncoordinated checkpointing and localized restarts [21, 22, 47]. Similarly, application specific recovery reconstructs lost data from the remaining correct data [1].

Most recovery schemes assume that a corruption is limited to a subset of values - e.g., values in the memory of one compute node. This condition is easy to satisfy with fail-stop failures, but may not be valid in the case of SDC detection with high latency. One solution is to verify all state outside of a corrupted subset [14]. However, such 
checks are expensive. An alternative is to determine the propagation of corruption when a detection occurs and to restrict recovery to the potentially impacted state. In this case, some corruption can be ignored as it is attenuated by the algorithm; in other scenarios, corrupted values may permanently influence the solution.

Prior work has explored how deviations from bit-flips in floatingpoint computation impact on convergence properties [16, 17]. Other work has used corruption propagation in the development of lowlevel instruction based detection schemes that check invariants or create SDC detectors inside the compiler [23, 38] or utilize code replication to detect corruption in instructions that are likely lead to and propagate corrupted state $[18,27,28]$, and understanding long latency crashes $[30,45]$. This paper combines the latency of programmatic systems of corruption - e.g., segfaults, detection, control flow divergence, with corruption propagation in state variables to discuss the impact of detection latency on recovery options. In the context of tracking corruption propagation inside applications, [2] looks at state corruption propagation in MPI codes by tracking number of incorrect memory addresses, but does not relate corruption back to application level data structure nor explore compiler optimizations and the impact of local problem size on corruption propagation. [31] quantifies corruption in different GPU and host memories in GPGPU programs. This papers focus on MPI applications and tracks corruption in state variables and across MPI processes.

A detailed view of corruption propagation offers a measure of an application's ability to withstand soft errors. Moreover, it also helps application developers identify where to place detectors and to identify locations for data recovery. This paper makes the following contributions:

- the impact of detection latency on recovery options;

- the data structures critical to corruption and the risk of obtaining invalid results;

- the influence of compiler optimizations on state corruption propagation; and

- the analysis of problem size and inter-node corruption propagation.

The remainder of this paper is structured as follows. Section 2 discusses corruption propagation for sparse matrix-vector multiplication and motivates the need to investigate state corruption propagation in application codes. Section 3 details the design and overview of the corruption propagation tool and how it tracks propagation at a micro and macro-level. Error propagation results are presented in Section 4 along with a discussion on detection and recovery options. Related work is discussed in Section 5.

\section{MOTIVATION}

\subsection{Sparse Matrix-Vector Multiplication}

Sparse matrix-vector multiplication (SpMV) is the core compute kernel in many HPC applications. To understand corruption propagation, it is helpful to view a SpMV as of series of inner-products between the rows of the matrix and the input vector or more simply as an unstructured series of multiplications and additions.

In Figure 1, one element of the input vector is corrupted (red square). The corrupted value spreads through three inner products (rows), resulting in the corruption (red squares) in the output vector.
If this SpMV is used as part of an iterative method or solver, then the corrupted values propagate further as the output vector is used in other parts of the method and with repeated application of the SpMV. Consequently, corruption in element $i$ of the input vector influences element $j$ of the output vector if row $j$ in column $i$ is non-zero. Thus, the propagation of corrupted values with dense matrices is more rapid than with sparse matrices [13].

$$
\left(\begin{array}{ccccc}
x & x & & \\
x & x & x & & \\
& x & x & x & \\
& & x & x & x \\
& & & x & x
\end{array}\right)(\square)=\left(\begin{array}{l}
\square \\
\square
\end{array}\right)
$$

Figure 1: Propagation of corrupted state via a sparse matrixvector multiplication.

One important caveat of corruption propagation for a SpMV is that corruption is modeled through the algorithm and not by the code as executed on a computer. A complete analysis of corruption propagation for the SpMV requires investigating the effect of corruptions of loads, stores, address calculations, branching, looping, etc. This is often complex as deep inspection of the code is needed in order consider all paths of execution. The corruption propagation tool presented in this paper allows for tracking the propagation of corruption at the level of application variables and with load/store granularity.

\section{TRACKING PROPAGATION}

Figure 2 details the flow of a fault occurring in a system that transitions to a failure. Once a fault is activated, error is present in the system. In this paper, error resulting from an activated fault is referred to as state corruption or corruption to avoid confusion with numerical errors in HPC applications. The location of the initial corrupted value is critical to identifying corruption propagation. During the execution the remaining program instructions, the corruption can be masked due to programmatic or algorithmic properties, can lead to a system detectable event such as a segmentation fault, or can propagate to corrupt more of the program state and become a silent data corruption (SDC). SDC detection schemes rely on a portion of the application state being corrupted, or a large magnitude in the deviation to detect simulation divergence.

Two levels of corruption propagation are considered:

macro tracks deviations in the state variables through the simulation; and

micro tracks deviations in the loads, stores, and other low-level operations.

In order to track corruption propagation at both the micro and macro level, an LLVM-based [29] instrumentation tool is developed to emulate lock-step execution of a correctly executed gold

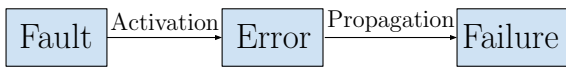

Figure 2: Logical flow of error propagation from initial fault to subsequent failure. 


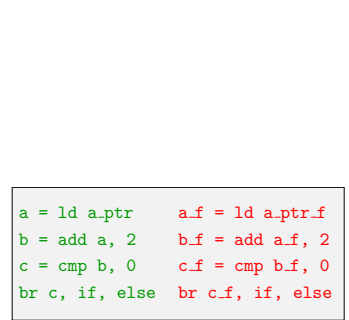

(a) Code is duplicated forming two sets gold (green code) and faulty (red code).

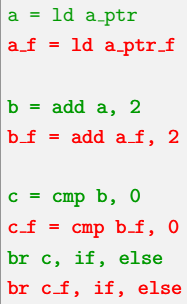

(b) gold and faulty code is interleaved to emulate lock-step execution.

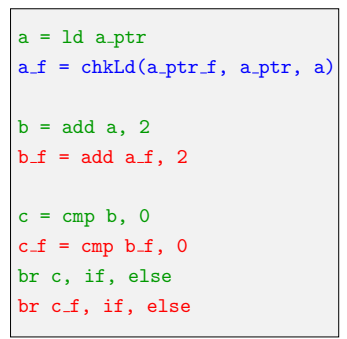

(c) All loads and stores in faulty are replaced with instrumentation calls to tation call to check for contrack corruption propaga- trol flow divergence. tion at a load/store level of accuracy. with an instrumen-
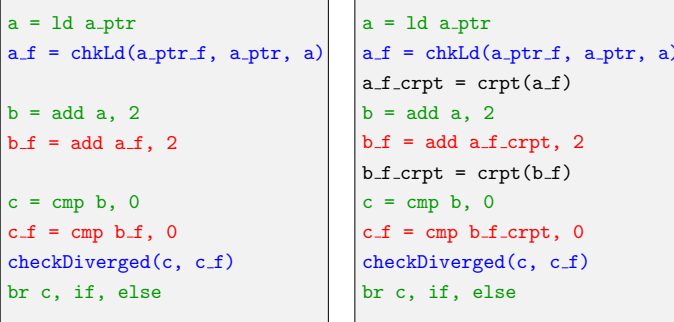

(e) faulty instructions are run though the fault injector FlipIt [11] to instrument them for fault injection.

Figure 4: Overview of the code transformation for micro-level propagation tracking.

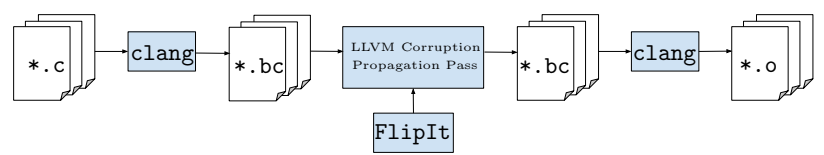

Figure 3: Overview of the instrumentation process that transforms source code to be able to track propagation and inject faults.

along with a faulty execution faulty. Optimized LLVM IR code is run though a compiler pass to instrument it to track corruption propagation and to inject faults before being compiled to object code - see Figure 3. Figure 4 illustrates the instrumentation process performed in the compiler pass. Source code is instrumented (Section 3.1) after compiler optimizations are performed to mitigate the impact of instrumentation on the object code generated. An API call at natural points in the application - e.g., end of an iteration - tracks propagation of application level memory allocations at the macro-level. At the micro-level, the LLVM compiler pass adds instrumentation to track propagation at a load/store granularity and relates loads/stores to application level memory allocations.

After replication, there exists two distinct sets of instructions: one for the gold application (green) and one for the faulty application (red). Instructions for the gold application forms a reference set of loads, stores, and correct program behavior that the faulty application instructions are compared against. Replicating all instructions creates separate memory images for both the gold and faulty applications. Thus, these two sets of instructions do not share the same data.

\subsection{Micro-level Propagation Tracking}

At the micro-level, loads and stores are monitored for deviations since these operations directly impact the state variables. Stores commit erroneous values to state variables, while loads allow for reuse and propagation of corrupted data. Examining corruption propagation at the micro-level allows a fine grain view of how corruption propagates inside the application. This is useful to assess the bound on a pointer reference leading to a segmentation fault, to determine the number of loads and stores that are perturbed, to quantify the deviation when a corruption is masked by the application, and to examine the impact of compiler optimizations at a fine-grain level.

Because instructions from gold and faulty are interleaved, corresponding operations - i.e. loads and stores - are next to each other allowing for straightforward comparison of both the values and the addresses. To simplify the comparison, all loads and stores in faulty are replaced with an instrumentation call, see Figure 4c. In addition to determining if a loaded or stored value deviates from the expected gold value, the deviation can be calculated (with additional computational cost) along with information on the associated allocation. The latter requires the base address and size of all memory allocations to be logged by instrumenting memory allocation calls and by inserting the base address and size into a table. Information that relates the gold and faulty allocations is also logged since it is used to assess macro-level propagation.

Algorithms 1 and 2 detail the logic of the instrumentation calls for loads and stores, respectively. The function call to checkInMemory determines whether an address is contained in a logged memory allocation of the faulty application. The information collected inside the function logDeviation of Algorithm 3 accumulates information into the same variables for both loads and stores; however, in practice a vector of statistics exists for loads and stores. If a store is outside an allocated memory region of the faulty application, then the address and value are added into an outside store hash table to allow subsequent loads to read the incorrectly written value and to prevent overwriting of data used by the gold application. Conversely, if a load from faulty occurs outside an allocated memory region, the load proceeds only if the address does not exist in the outside store hash table. If the address is found in the outside store hash table, the faulty address is de-referenced in an effort to generate a segmentation fault, but execution proceeds with the value from the store hash table.

Once a fault is injected, it propagates to registers and memory locations based on data-dependencies. Periodically, these datadependencies flow to a comparison used in a branch. Control flow 

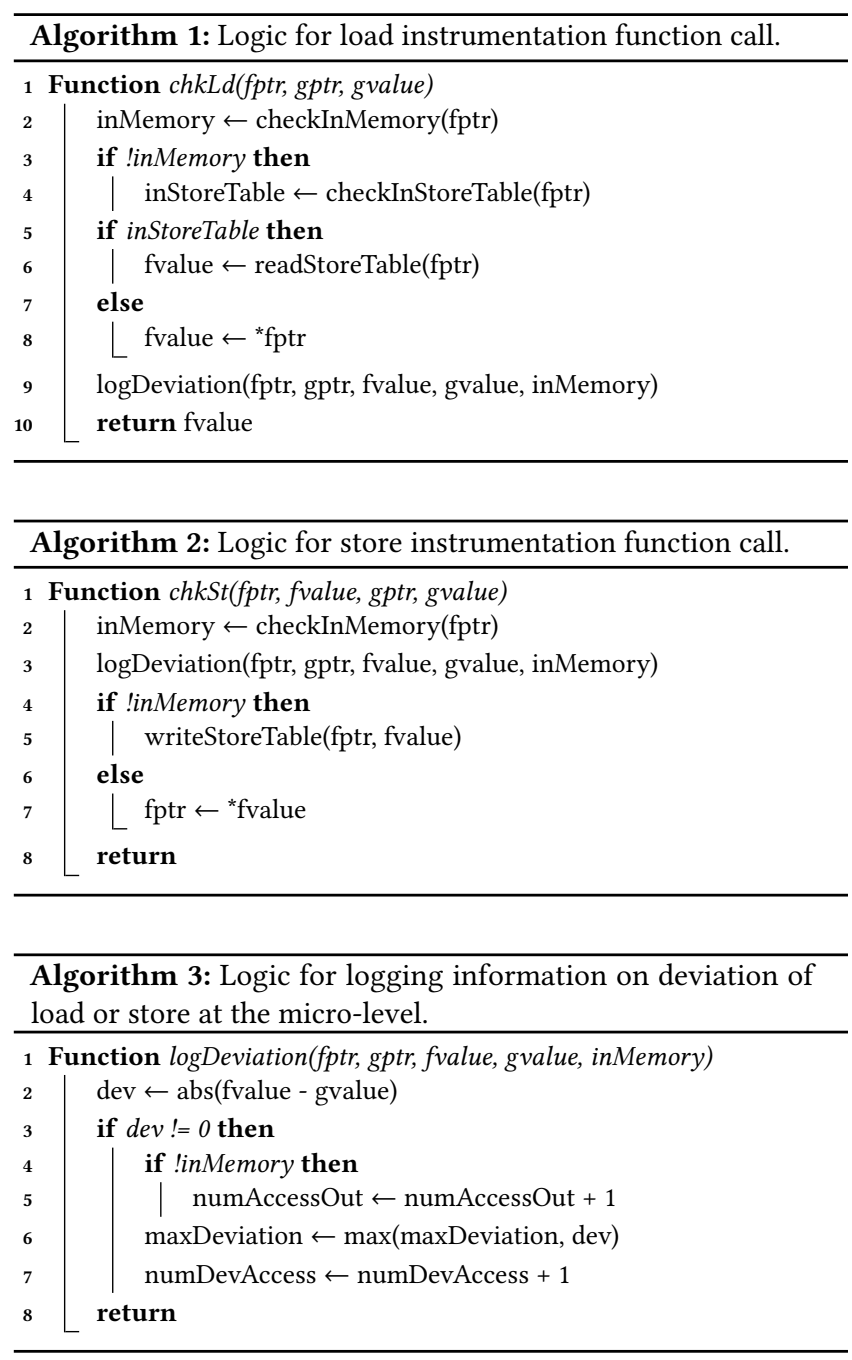

in the lock-step execution relies on the values from the gold application - see Figure 4d. Comparisons in faulty remain, but the branching instruction is replaced with an instrumentation call to log information about this branching deviation. Due to lock-step style execution, micro-level propagation tracking is unable to accurately resolve control flow divergence. Resolving this requires a coarser view of propagation tracking: macro-propagation tracking.

To resolve control flow divergence and to handle regions of code not open to instrumentation - e.g., MPI_Send, MPI_Recv - function calls are duplicated. For functions that modify global state and yield a different result when called by both the gold and faulty code - e.g., rand(), MPI_Init () - the return value is duplicated allowing both the gold and faulty codes to proceed with the intended value/action.

For functions open to instrumentation, the LLVM pass creates a new version of that function with an extended interface. The function's argument list is duplicated providing arguments for the gold and faulty code. In addition, the return type is modified to return a structure containing two elements: the return value for both the gold and the faulty code. Code contained inside the original function is duplicated and combined as shown in Figure 4. faulty code depends on and consumes the faulty arguments, and the gold code depends on and consumes the gold arguments.

After the gold and faulty code has been merged and all instrumentation is complete, faulty instructions are passed to FlipIt [11], an LLVM based fault injector, to instrument the faulty instructions for fault injection - see Figure 4e. For instructions that have been replaced with instrumentation calls, FlipIt is instructed to only inject faults in arguments belonging to the faulty code. Arguments coming from gold will never suffer fault injection in instrumentation calls.

\subsection{Macro-level Propagation Tracking}

Macro-level propagation tracking targets the deviation of high-level state variables, data structures, and propagation across process boundaries. To facilitate tracking propagation of corruption in state variables, the base address of all memory allocations along with the allocation's length are stored in a table. During compilation, the LLVM corruption propagation pass inserts an instrumentation call after the memory allocation in the gold and faulty code that logs both allocations into the table. Furthermore, this instrumentation call creates a relationship between the two allocations allowing for comparison of indices when computing propagation statistics. Algorithm 4 details the logic used when comparing two memory allocations. By default, only the percent of elements corrupted, $\ell_{2}$-norm, and max-norm between the gold and faulty memory allocations is logged for post run analysis.

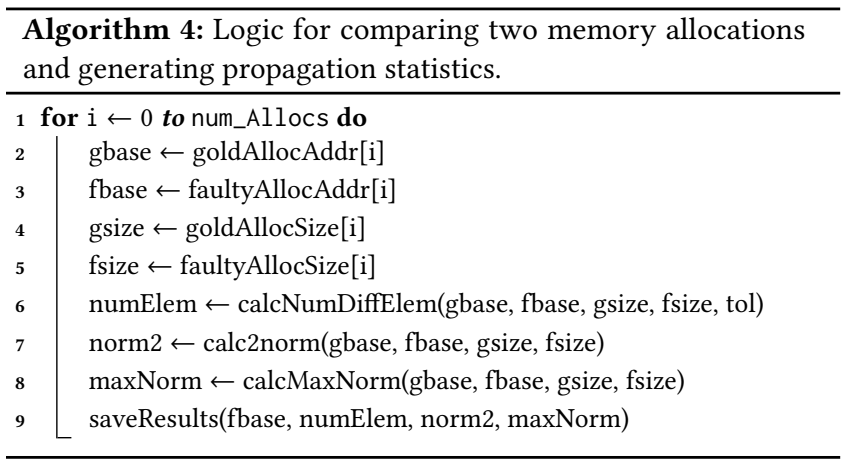

Comparing the state of all allocated variables is expensive; therefore, comparison points are placed at natural termination points e.g., end of an iteration. These locations correspond to locations where SDC checks are often placed and checkpointing occurs. Initially, corruption is confined to the process in which the fault occurred. As the application progresses, inter-process communication allows corruption to propagate beyond the process suffering the fault. Understanding how fast this occurs and which processes are likely to have corrupted data allows for only a subset of processes to recover by checkpoint-restart or a more tailored algorithmic solution.

Unlike micro-level propagation tracking, macro-level propagation tracking is able to resolve divergence in control flow graph by executing functions to completion once for the gold arguments and lastly for the faulty arguments. Control flow may diverge inside the function, but at the point the function call returns both the gold 
and faulty applications are at the same point in the control flow graph. Thus, comparing the states of the gold and faulty memory allocations is safe and meaningful.

\section{EXPERIMENTAL RESULTS}

\subsection{Testing Methodology}

4.1.1 System. Results are collected on Blue Waters, a Cray supercomputer managed by the National Center for Supercomputing Applications and supported by the National Science Foundation and the University of Illinois. Each compute node has 2 AMD 6276 Interlagos CPUs and 64 GB of RAM. Clang and LLVM version 3.5.2 compile and instrument the source code.

4.1.2 Fault Injection. The fault model used in this paper assumes that transient faults arise in processor logic during execution. That is, the fault manifests as a single bit-flip in the result register of the instruction. In addition, it is assumed that register files and memories such as SRAM and DRAM are sufficiently protected by error correcting codes (ECC) or more advance features such as Chipkill [15], and that faults are not injected in these locations. The LLVM corruption propagation pass uses the open-source LLVM based fault injector FlipIt [11] to instrument instruction from the faulty application, allowing for faults to be injected dynamically at runtime.

This paper does not inject faults into the initialization of the applications. Instead, faults are injected during the main computation. The selected applications use MPI, and in the tests, MPI process rank 3 is selected to experience a single bit-flip error during the execution of a unique, random dynamic LLVM instruction. Instructions selected for fault injection are classified into the following categories based on its use in code: floating-point arithmetic (Arith$F P$ ), fixed-point integer arithmetic (Arith-Fix), pointer and address calculation (Pointer), and branching, comparisons, and control flow (Control). Latency, in number of instructions, counts dynamically executed LLVM instructions.

Although a single MPI process suffers a fault, all MPI processes still track propagation via the methods outlined in Section 3 in order to determine if propagation occurs between nodes.

Each application is run 1500 times with a different random fault each time. The kernels WAXPY and SpMV are run 1500 times per optimization level each with a different random fault.

4.1.3 Applications. Jacobi: This defines Jacobi relaxation on a unit square with fixed boundaries using a 5-point stencil and 1-D row partitioning of parallel processes. This test uses 4 MPI processes with 4096 grid points per process. Instrumentation yields a $102 \times$ slowdown. Jacobi relaxation does not guarantee a reduction in the residual, however unexpected large jumps often indicate the presence of state corruption. This paper flags any increase in the residual by an order of magnitude as SDC. The stopping tolerance use for Jacobi is $1 e-3$, and the floating-point comparison tolerance when tracking propagation is set to $1 e-5$. Finally, macrolevel propagation results are logged at the end of each iteration.

CoMD: CoMD $^{1}$ is a molecular dynamics mini-app created and maintained by the Exascale Co-Design Center for Materials in Extreme Environments (ExMatEx). CoMD is parallelized with MPI

\footnotetext{
${ }^{1}$ https://github.com/exmatex/CoMD
}

and uses a link-cell structure to determine the interaction regions for the atoms. This test uses $16 \mathrm{MPI}$ processes to simulate the motion and interaction of 32000 atoms over 500 time-steps, where forces between atoms are computed using the Embedded-Atom Method (EAM). Instrumentation results in a $33 \times$ slowdown and SDC is detected by ensuring that the total energy is within five standard deviations of the ensemble mean. Macro-level propagation results are logged at the end of each iteration. Deviations smaller than $1 e-10$ are considered insignificant to accuracy of CoMD.

HPCCG: $\mathrm{HPCCG}^{2}$ is a conjugate gradient (CG) benchmark from the Mantevo Suite that simulates a 3D chimney domain using a 27point finite difference matrix. This test is run with 16 MPI processes and a local block size of $n x=n y=n z=13$ and instrumentation results in a $460 \times$ slowdown. As with Jacobi, the CG algorithm does not guarantee a reduction in the residual. Here, any increase in the residual by an order of magnitude is flagged as SDC. Macro-level propagation results are logged at the end of each iteration. The convergence tolerance used for HPCCG is $1 e-7-$ when tracking corruption propagation, deviations smaller than $1 e-10$ are ignored.

\subsection{Micro-level Propagation}

4.2.1 Latency of Detection. Each injected fault starts as a singlebit error in the result register of an instruction. As the program executes, the single-bit error propagates to other registers and memory locations. Depending on the type of instruction the fault is injected into, the latency to detection often varies. The analysis in this paper looks at three triggers to failure: segmentation fault, detection, and control flow divergence. Table 1 shows the breakdown of each symptom based on instruction type across the tests in Section 4.1.3.

Segmentation faults account for $30-35 \%$ of all injected faults in each application. From Table 1, the majority (over 50\%) of segmentation faults are triggered by Pointer instructions. Figure 5 shows the segmentation fault latency for each application in number of LLVM instructions executed after an injection. A significant number $(90 \%)$ of segmentation faults occur within 4 LLVM instructions. Of the runs with a segmentation fault within 4 LLVM instructions, $61 \%$ percent are classified as Pointer, $32 \%$ percent are classified as Arith-Fix, 0\% percent are classified as Arith-FP, and 13\% percent are classified as Control. Looking at the remaining segmentation faults, $16 \%$ percent are classified as Pointer, $8 \%$ percent are classified as Arith-Fix, 0\% percent are classified as Arith-FP, and 76\% percent are classified as Control. Short latencies are attributed to corruption of address calculation - e.g., corrupting an address or offset, before a load or a store. Longer latencies are the result of corruption in the loop induction variables. The instructions used to check a loop conditional increases the segmentation fault latency slightly before the induction variable is used as part of a load/store during the subsequent iteration. Segmentation fault latencies for these applications are consistent with those reported for the Linux kernel [20] and other benchmarks [30].

Figure 6 shows the bit locations that generated a segmentation fault. The results highlight that most segmentation faults occur due to a bit-flip near the most-significant bit (MSB). Bit-flips in bits near the least-significant bit (LSB) lead to incorrect indexing and using

\footnotetext{
${ }^{2}$ https://mantevo.org/packages.php
} 
Table 1: Breakdown of failure symptom by instruction type.

\begin{tabular}{cccc} 
Instruction & \multicolumn{3}{c}{ Failure Symptom } \\
Type & $\begin{array}{c}\text { Segmentation } \\
\text { Fault }\end{array}$ & Detection & $\begin{array}{c}\text { Control Flow } \\
\text { Divergence }\end{array}$ \\
\hline Arith-FP & $0 \%$ & $49 \%$ & $20 \%$ \\
Arith-Fix & $24 \%$ & $24 \%$ & $26 \%$ \\
Pointer & $56 \%$ & $27 \%$ & $16 \%$ \\
Control & $20 \%$ & $0 \%$ & $38 \%$ \\
\hline
\end{tabular}

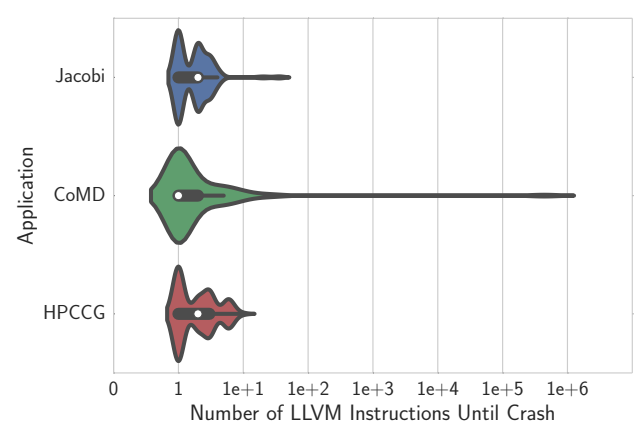

Figure 5: Latency (number of LLVM instructions) until a segmentation fault.

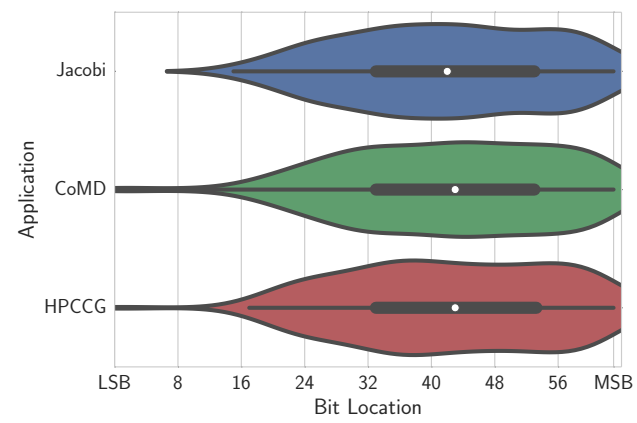

Figure 6: Bit positions where injected fault resulted in a segmentation fault.

incorrect data. Flipping bits $0-2$ result in unaligned data access for double precision arrays and can cause significant deviation in the load/store, but rarely generates a segmentation fault. As the local problem size grows, the amount of allocated memory also increases. As a result, the number of segmentation faults decreases for low order bits by replacing them with load/store on incorrect addresses.

Segmentation faults are an excellent detector that allow little corruption propagation in most cases. Since segmentation faults occur in only $30-35 \%$ of the tests most faults allow for propagation and SDC. Typical HPC SDC detectors check for errors at a coarse granularity (thousands or millions of instructions) by looking for

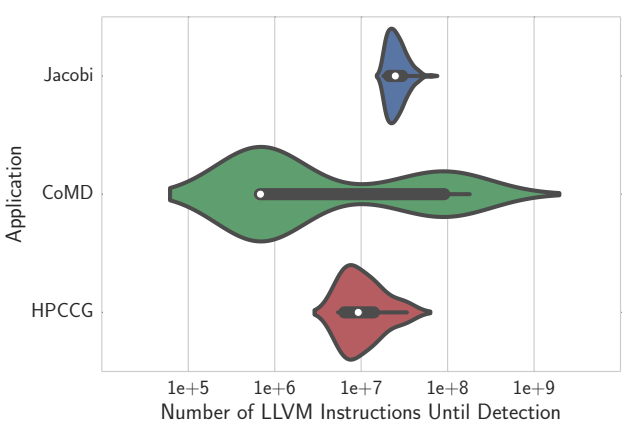

Figure 7: Latency (number of LLVM instructions) until SDC detection.

latent errors that have corrupted the state of application level variables to cause a noticeable deviation. Overall the lightweight SDC detectors added to tests (Section 4.1.3) detect data corruption in $11 \%$ of Jacobi runs, $1 \%$ of HPCCG runs, and $2 \%$ of CoMD runs. Breaking down which faults are detected by instruction type, Table 1 , shows most detected faults occur in Arith-FP instructions. Figure 7 shows the latency in number of LLVM instructions executed before the detection occurs. Compared to detection via segmentation faults, detection latency of specially designed detectors is much larger.

Each test checks for SDC at the end of each iteration. Converting detection latency from LLVM instructions executed to iterations executed shows all runs with detection for CoMD and HPCCG are within 2 iterations of injection. Detected SDC in Jacobi has a longer maximal latency at 492 iterations. Faults that cause an order of magnitude differences in floating-point values are detected during the following iteration. Without SDC detectors $100 \%$ of HPCCG runs and $56 \%$ of Jacobi would require extra iterations to converge to the correct solution. All CoMD runs with detection appear as outliers when forming an ensemble distribution at the final time-step if no SDC detectors are present.

If checkpoint-restart routines are used to recover from the detected SDC, then for runs with a high latency of detection, a domino effect of needing to roll back older and older checkpoints is possible if recovery proceeds from frequently taken in-memory checkpoints; this is common for multi-level checkpointing schemes [7,35]. At the time of detection, if the extent of corruption is known in terms of state variables and processes, then forward-recovery schemes offer the best solution to avoid the domino effect of checkpoint-restart (backward-recovery).

4.2.2 Abnormal Behavior. HPC applications commonly iterate over and compute on vectors of data. Faults in Pointer and Arith-Fix instructions often lead to corruption in pointers used in load/store operations, and can lead to segmentation faults. However, corruption of loop control variables can lead to divergence in the control flow graph. Control flow divergence in loops (see Figure 8) results in an early loop exit; consequently, elements of a vector may not be updated or computed. Control flow divergence occurs in $3 \%$ of Jacobi runs, $6 \%$ of HPCCG runs, and $20 \%$ of CoMD runs. Unlike HPCCG and Jacobi where data distribution is static, CoMD has atoms that migrate between processes that dynamically modify the 


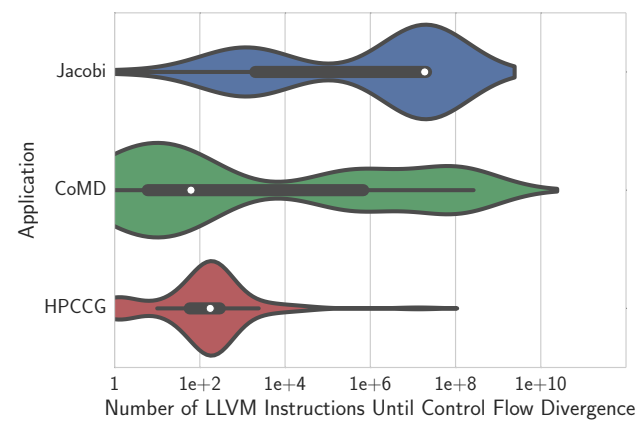

Figure 8: Latency (number of LLVM instructions) until control flow diverges.

Table 2: Dynamic LLVM instruction type percentage

\begin{tabular}{cccc} 
& \multicolumn{3}{c}{ Application } \\
Instruction Type & HPCCG & Jacobi & CoMD \\
\hline Arith-FP & $35 \%$ & $41 \%$ & $35 \%$ \\
Arith-Fix & $22 \%$ & $28 \%$ & $16 \%$ \\
Pointer & $22 \%$ & $21 \%$ & $34 \%$ \\
Control & $21 \%$ & $10 \%$ & $15 \%$ \\
\hline
\end{tabular}

data distribution. The extreme latencies for control flow divergence in CoMD are due to corruption of the atom positions that modifies the control flow of the atom exchange routine as atoms migrate incorrectly (inter-process corruption propagation). In the remaining cases, most control flow diverges within the injected loop structure, leaving some vector entries unmodified. To ensure correct execution of these loops, instruction duplication techniques such as IPAS [28] and FlipBack [36] or low-cost invariant checks [23], offer the ability to ensure correct control flow with minor overheads.

An optimization to lower the overhead of algorithmic based SDC detection schemes is to assume static data - e.g., the matrix in a linear solver is sufficiently protected that it does not need explicit checks for consistency and leaving SDC checks to inspect dynamic data. With a fault model that only allows for corruption in Arith-FP values, this is a valid assumption, however once corruption occur in Arith-Fix and Pointer type instructions, static data can be written by errant store instructions. Although very rare (less than $0.1 \%$ of runs) it does have the ability to make a convergent algorithm non-convergent or converge to a different solution. To mitigate corruption of static data, checksums can be employed to ensure consistency or pages containing static data can be marked read-only after initialization to ensure no errant stores corrupt the data.

4.2.3 Effect of Compiler Optimizations. The ratios of the different instruction types impact the probability of different failure symptoms. Table 2 shows the percentage of dynamic LLVM instructions for each application classified as a given instruction type. Across all applications, instructions classified as Arith-FP comprises the majority dynamic instructions followed by instruction types Pointer and Arith-Fix that are used to compute addresses. Finally, Control flow makes up the smallest classification percentage.
Instruction mix depends on the data structures, compiler, and optimization level. Two key operations in the HPCCG mini-app and other linear solvers are the sparse matrix-vector multiply (SpMV) and scaled vector addition (WAXPBY). The impact of compiler optimizations on these small kernels helps identify the impact on the full mini-app and production application.

WAXPY: A WAXPY operation, Algorithm 5 scales two input vectors $\mathbf{x}$ and $\mathbf{y}$ when performing the vector addition operation. Compiling without any optimizations $(-00)$ produces verbose and explicit code as every load and store references memory. Register allocating variables $\mathrm{i}$ and $\mathrm{N}$ along with hoisting loads outside the loop with -01 reduces address calculation and loads/stores that often lead to segmentation faults, see Section 4.2. Furthermore, optimization levels -02 and -03 unroll and vectorize the loop, further reducing the need for control flow instructions. For this kernel, -02 and -03 produces identical code. To support higher level optimizations such as loop unrolling and vectorization, extra instructions are added to ensure correctness for all sizes of $\mathrm{N}$. Table 4 summarizes the impact of compiler optimizations. Vectorization increases the number of integer operations which causes an increase in runs that experience a segmentation fault. Loop unrolling removes branching instructions and instructions that update the loop induction variable which removes locations where corruption of loop induction variables are possible lowering control flow divergence.

\begin{tabular}{l}
\hline Algorithm 5: Scaled Vector Addition (WAXPBY). \\
\hline 1 for $i \leftarrow 0$ to $N$ do \\
$2 \quad\left[w[i] \leftarrow a^{*} x[i]+b^{*} y[i]\right.$ \\
\hline
\end{tabular}

Table 3: Dynamic LLVM instruction percentage for WAXPBY kernel.

\begin{tabular}{ccccc} 
Optimizations & Arith-Fix & Pointer & Control & Arith-FP \\
\hline O0 & $31 \%$ & $23 \%$ & $15 \%$ & $31 \%$ \\
O1 & $8 \%$ & $23 \%$ & $23 \%$ & $46 \%$ \\
O2/O3 & $17 \%$ & $40 \%$ & $3 \%$ & $40 \%$ \\
\hline
\end{tabular}

SpMV: The SpMV kernel, Algorithm 6, is more complicated than that of WAXPBY both mathematically and in machine code. Because the SpMV uses a sparse matrix representation (compressed sparse row in Algorithm 6), the level of indirection needed to access entries of the matrix increases. Each increase in indirection involves a pointer dereference; therefore, with more address manipulations and loads/stores, the number of dynamic instructions of Arith-Fix and Pointer are higher than with WAXPBY, as shown in Table 5. This

Table 4: WAXPBY kernel failure symptom percentage.

\begin{tabular}{cccc} 
& \multicolumn{3}{c}{ Optimizations } \\
Symptom & O0 & O1 & O2/O3 \\
\hline Segmentation Fault & $29 \%$ & $27 \%$ & $40 \%$ \\
Control Flow Divergence & $10 \%$ & $4 \%$ & $2 \%$ \\
No Symptom & $61 \%$ & $69 \%$ & $58 \%$ \\
\hline
\end{tabular}


also implies that common symptoms of these types of operations will be more prevalent.

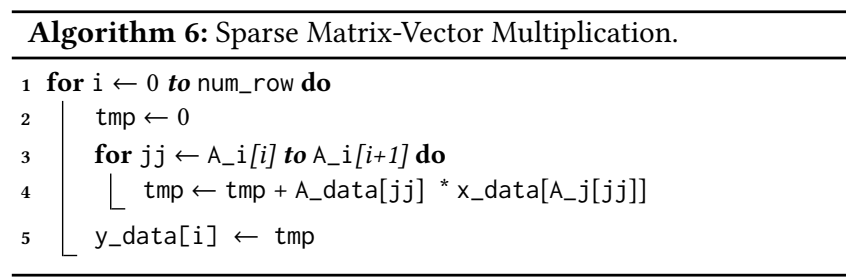

With the baseline optimization level -00 , the code is explicit and verbose. Higher levels of optimizations retain the base addresses of the arrays $A_{-} i, A_{-} j, A_{-}$data in register temporaries along with loop induction variables and hoists loads with -01 . Optimization levels -02 and -03 do not vectorize this kernel. Instead, the inner most loop is unrolled removing comparisons and branching instructions along with hoisting the load of num_row to outside the loops.

Table 6 shows that without vectorization, the rate of segmentation faults remains around $30 \%$ of executions, which is consistent with WAXPBY and the applications. Loop unrolling fails to reduce divergence in control flow compared to WAXPBY. Control flow for the inner most loop is more complex than with WAXPBY resulting in more locations in which a fault can occur that influences control flow.

\subsection{Macro-level Propagation}

After a fault occurs, an erroneous value is present in the application state. Over time, as this value is used/reused, the corruption propagates to infect larger portions of the application state. Most HPC SDC detectors ensure correctness of application level variables. Knowing which application variables are corrupted and the extent of corruption can assist in placement of detection and recovery schemes.

4.3.1 Jacobi. Each iteration of Jacobi refines a solution $u$ to improve the solution accuracy resulting in an updated solution in a separate vector $u_{n e w}$. These two vectors represent the two key data structures and is the focus when measuring corruption

Table 5: Dynamic LLVM instruction classification percentage for SpMV kernel.

\begin{tabular}{ccccc} 
Optimizations & Arith-Fix & Pointer & Control & Arith-FP \\
\hline O0 & $38 \%$ & $40 \%$ & $6 \%$ & $16 \%$ \\
O1 & $22 \%$ & $24 \%$ & $21 \%$ & $33 \%$ \\
O2/O3 & $24 \%$ & $23 \%$ & $19 \%$ & $34 \%$ \\
\hline
\end{tabular}

Table 6: SpMV kernel failure symptom percentage.

\begin{tabular}{cccc} 
& \multicolumn{3}{c}{ Optimizations } \\
Symptom & O0 & O1 & O2/O3 \\
\hline Segmentation Fault & $25 \%$ & $32 \%$ & $29 \%$ \\
Control Flow Divergence & $12 \%$ & $13 \%$ & $14 \%$ \\
No Symptom & $63 \%$ & $55 \%$ & $57 \%$ \\
\hline
\end{tabular}

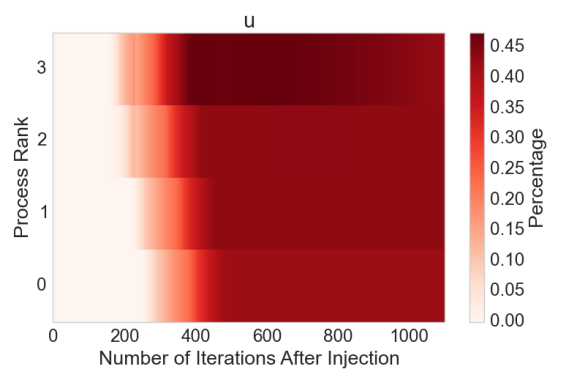

Figure 9: Average percentage of corrupted elements of iterative solution $u$ for Jacobi.

propagation. Figure 9 shows the average percentage of elements that are corrupted due to an injected fault (intensity of color) across all MPI processes ( $y$-axis) in the iterations following the injection iteration ( $x$-axis) for the solution variable $u$. The variable $u_{n e w}$ is not shown as it is qualitatively similar to $u$. All runs are aggregated to align the iteration where injection occurs. This allows corruption percentages in all remaining iterations to be averaged over all runs. All faults are injected on rank 3 , and as time evolves corruption propagation occurs inside this process indicated by the increase in the intensity of color on the horizontal row for rank 3. Overtime, corruption propagates inside rank 3 and reaches the region of the array that is communicated via a halo-exchange corrupting process 2. This process continues until all processes are corrupted or the corruption is attenuated. Because Jacobi converges to a solution, over time the corruption in the variables is removed. When tracking propagation in $u$, comparisons are made between the memory of $u$ from the gold and faulty applications. As Jacobi continues to iterate, error does not appear to reduce because as error due to the fault is removed from the faulty $u$ it is being compared to an ever more accurate $u$ from the gold application. Only with extra iterations on faulty (beyond what is run for gold) do the two solution vectors converge.

After a fault, corruption appears immediately on rank 3. As corruption propagates inside rank 3 , it corrupts values set to process 2 in a halo exchange the speed of this corruption depends on the stencil size. This problem uses a 5-point stencil, and the average worst case propagation latency occurs when an element interior to a local domain is corrupted. This requires $n / 2$ iterations were $n$ is the local block size. SDC detected in Jacobi is within 492 iterations of injection. For any reasonable local block size, once SDC is detected it can be confined to a process and its immediate neighbors; allowing a customized local recovery scheme to be applied.

4.3.2 HPCCG. Conjugate Gradient (CG) - see Algorithm 7 - is a popular solver for systems of linear equations. This algorithm relies on four key variables: the iterative solution $x^{k}$, search directions $p^{k}$ that are used to update $x^{k}$, residual vector $r^{k}$, and the matrix-vector product $A * p$.

As with Jacobi, propagation results for HPCCG are aggregated to align the iteration in which injection occurs. Figure 11 shows corruption propagation in the form of the average percentage of 


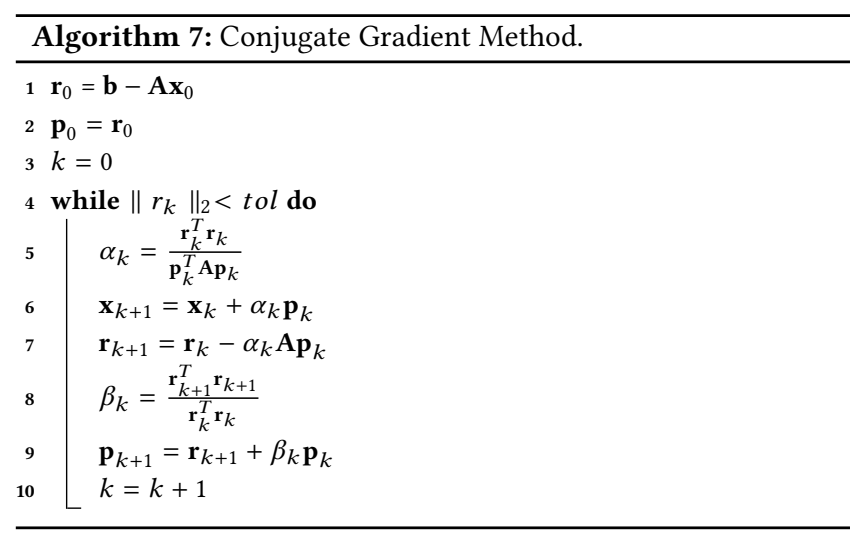

elements corrupted (color) per variable across all MPI processes ( $y$ axis) for subsequent iterations after injection ( $x$-axis). As corruption propagates locally inside each variable, the horizontal color for that row grows darker. As corruption is removed, the color lightens. Propagation between processes can be seen by looking at the color progression of columns at each iteration.

Initially, the average percentage of elements corrupted in each vector is small. However, as HPCCG continues to iterate, corruption begins to propagate both internally and externally of the corrupted process, rank 3 . The most severe corruption is confined to rank 3 and the neighboring processes across the majority of the iterations. Dependencies between the four variables in Figure 11 are due a corruption in the variables $r, p$, or $A p$, which leads to corruption in the solution vector $x$. Furthermore, the SpMV propagates corruption as shown in the corresponding corruption in $p$ and $A p$ on every processes of each iteration. Data dependencies in updating other vectors further propagates corruption in $A p$ to all other variables. Ensuring the correctness of $p$ limits corruption propagation from the SpMV in $A p$ and subsequently corruption propagating to $r$ and $x$.

To see corruption propagation between variables more closely, Figure 10 shows the average percentage of elements from each variable corrupted on rank 3 . As with Jacobi, the iterative solution $x$ increases in error initially, but over time does not appear to remove error due to soft error corruption. This error is reduced at each iteration, however the iteration does not converge back to gold $x$. Errors in $r$ closely follow those in $p$ as both are used in updating the other through a WAXPBY. As corruption in $p$ grows and subsides, $A p$ reflects and propagates the corruption accordingly.

The search direction $p$ is central to corruption propagation as it is used in updating the other variables. Ensuring that $p$ is computed correctly helps ensure that the other variables are computed correctly. Because CG uses inner-products, if masking does not occur, then corruption in input vectors propagates to all processes in one iteration. Therefore, some form of corruption is resident in all variables within 3 iterations for runs that did not produce a segmentation fault. Local recovery is still possible, though it is complicated by the presence of corruption on multiple processes.

4.3.3 CoMD. Molecular dynamics codes such as CoMD do not converge to the same solution with each execution of the program as with HPCCG and Jacobi. Instead, a single run is combined with

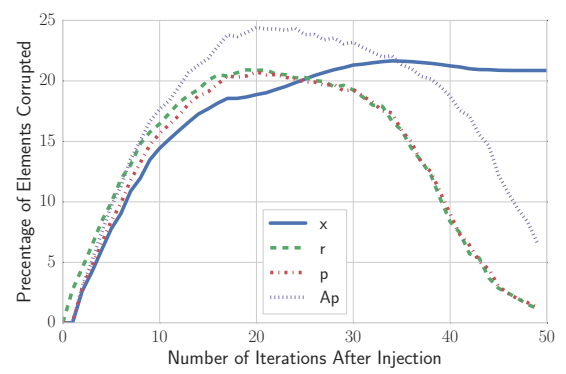

Figure 10: Average corruption in selected variables on rank 3 for HPCCG.

many other runs to form a statistical ensemble to analyze the distribution of key properties such as energy. This implies that small deviations can be masked if they do not modify the distribution. The key variables in CoMD for propagation analysis are: atom positions, atom momenta, atom forces, and atom energy. CoMD also differs from the other applications in how it stores its data. Because atoms migrate between processes, arrays are over allocated leaving space for other atoms from remote processes. This slack space is not contiguous within the arrays because it is allocated per local cell and not collectively for the entire local domain. The unused regions of the atoms complicate tracking propagation. Without modifying the data layout of CoMD this paper factors out the unused atom storage by using the number of atoms per process instead of the memory allocation size when computing corruption statistics.

Figure 12 shows the average percentage of corruption in the key variables of CoMD. For simplicity, only the force variable is shown. The remaining variables have a similar propagation pattern in terms of percentage of elements and ranks corrupted. Unlike HPCCG, where the SpMV rapidly propagates corruption between processes, the propagation in CoMD resembles Jacobi with corruption slowly propagating to neighboring domain regions. Although slow, the corruption propagation increases monotonically as time evolves which leads to an increased likelihood of the run becoming an outlier for large numbers of iterations. Over the iterations after an injection, $1 \%$ of the runs are classified as outliers when looking at the energy distribution at the final iteration. All of these outliers are caught by the SDC check.

The communication pattern in CoMD consists of point-to-point messages as atoms migrate from process to process. A single atom contributes to the force calculation of neighboring atoms. This region of influence is small and needs many iterations for the corruption to spread beyond the initially corrupted region of influence. This accounts for slow rate of propagation inside CoMD. Although corruption can propagate to neighbor processes, it requires tens to hundreds of iterations before corruption of an atom propagates to all processes. Corruption that impacts the simulation's energy distribution are detected within two iterations which allows for little inter-process propagation. Containment domains can be established around the process triggering the SDC detector and nearest neighbors to allow for partial recovery. 


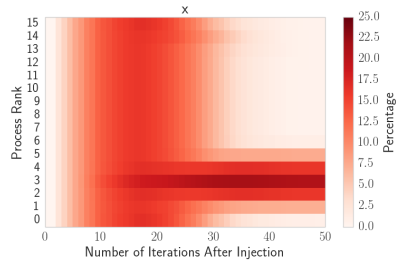

(a) Average percentage of corrupted vector elements for iterative solution $x$.

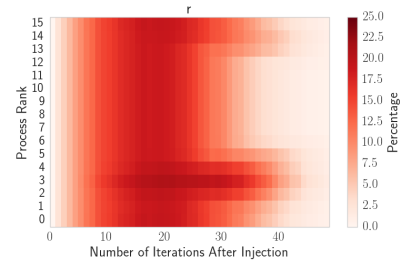

(b) Average percentage of cor- (c) rupted vector elements for residual $\mathbf{r}$.

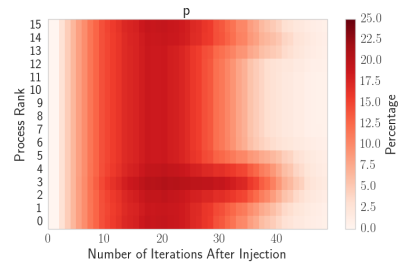

(c) Average percentage of corrupted vector elements for search directions $p$.

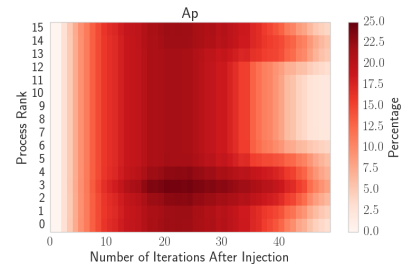

(d) Average percentage of corrupted vector elements for result of the SpMV of $A^{*} \mathbf{p}$.

Figure 11: Average percentage of corrupted vector elements for variables in the mini-app HPCCG in iterations after injection.

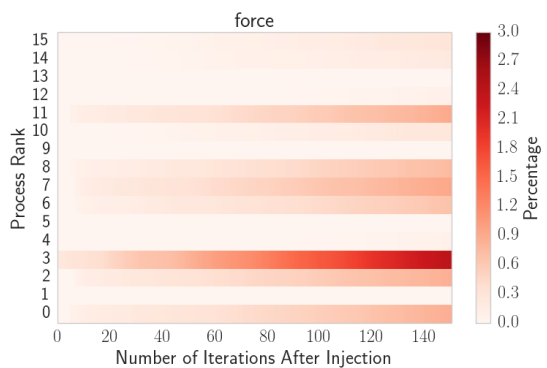

Figure 12: Average percentage of corrupted vector elements in the atom force array in the iterations following an injection for CoMD.

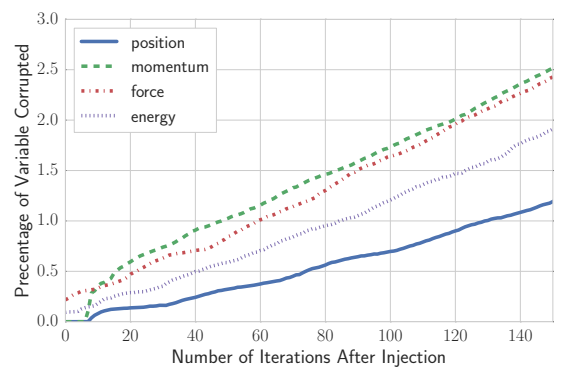

Figure 13: Average corruption in selected variables on rank 3 for CoMD.

4.3.4 Impact of Local Problem Size. To explore how problem size impacts corruption propagation. The preceding application experiments are re-run with the same number of MPI processes, but with more local work per process. Jacobi sees a $2.25 \times$ increase in the number of grid points per process at 36864 . The HPCCG local problem is $n x=n y=n z=16$, a $1.9 \times$ increase. Whereas CoMD is run with 62500 atoms, resulting in a $1.9 \times$ increase in problem size.

For Jacobi increasing the local problem size causes an increase in the average number of iterations to corrupt other processes by roughly $2 \times$. Corruption propagation patterns and failure symptoms resemble that shown previously. Increasing the local problem size for HPCCG sees no decrease in the speed of propagation to other ranks or a discernible change in failure symptoms. Due to the increased number of atoms in CoMD, more atoms fit into the same sized domain. When the force computation includes more atoms in the cutoff region, a single corrupted atom is now in more cut-off regions, thus propagating the corruption faster than previously observed. Beyond an increase in the propagation speed, failure symptoms for CoMD are comparable with previous results.

\section{RELATED WORK}

Redundancy: Redundancy is a common tool in detection schemes. Triple modular redundancy (TMR) [33] ensures correctness by computing values through triplicate and majority voting. Redundant threads or processes have been used to detect transient and permanent errors in hardware and software [19,37]. Redundancy at these levels often yield high overheads, but also high protection from soft errors. The GangES error simulator [41] seeks to limit the time required for fault injection experiments on applications running on micro-architectural simulators by checking for similarities in application state to prior fault injection runs. GangES does not consider parallel applications.

Tools: Corruption propagation modeling in static analysis combined with fault injection experiments is used extensively in compiler based protection schemes to identify the protection of key instructions. IPAS [28] leverages machine learning on fault injection experiments to learn which instructions are good candidates for replication to prevent program output corruption. However, this approach can be limited by a training dependence. Compiler analysis with profiling [27] can be used to improve the effectiveness of an instruction replication scheme; however the long term impact of corruption is not considered, such as between processes. SDCTune [32] uses static analysis, heuristics, and fault injection data to construct a probability model for corruption to decide what instructions are likely to generate SDC. Extensions of the Program Vulnerability Factor [18] metric have been used to distinguish between crashes and SDC allowing for more accurate estimates of SDC and crash rates. The modeling of corruption propagation is again limited to sequential programs. CrashFinder [30] explores the effectiveness of combining compiler analysis and fault injection results to identify instructions that generate long latency crashes due to pointer, loop, and state corruption; the propagation of corruption is not investigated. SymPLFIED [39] uses symbolic execution to 
abstract the state of corrupted values in the program to find hardto-detect fault injection locations that random fault-injection may reveal; this is limited to sequential integer programs.

Characterization: A study of the numerical impact of a single bit-flip on matrix-vector computations and GMRES is presented in [16, 17], respectively. An analysis of long latency crashes [45], shows corruptions in memory have long fault activation times leading to long latencies for crashes compared to corruption in registers, but does not investigate the extent of corruption propagation at the time of detection and the ability to recover locally. The impact of soft errors on the Linux kernel is determined [20], but does not quantify the corruption of system state variables to determine how to prevent propagation for long latency crashes. Y-branches [44] explores how control flow divergence impacts applications correctness and performance. Control flow re-converges if the architectural state exactly matches a golden copy; however, for applications that can remove/mask corruption over-time, this may underestimate the number Y-branches.

Characterizations of corruption in floating-point computation inside HPC applications is presented in [2]. Corruption is tracked based on terms of number of incorrect memory accesses and does not relate this to application level variables. A similar study [31], quantifies corruption in GPU and host memories for GPU benchmarks, but does not look at how corruption propagation in a distributed memory environment.

Recovery: Establishing a bound on the subset of total program state that is corrupted allows for localized recovery. Containment domains [14] surround code regions, using verification to ensure correctness. Identifying the location of code containment remains open. Similarly, transactional semantics [24] can be used for MPI, but not on application variables that may be corrupted. Esoftcheck [46], uses compiler analysis to remove redundant SDC detectors to maintain high reliability, but does not consider the latency of detection and how it effects propagation. An analytic version of this problem which investigates optimal placement of detectors of different capabilities to verify a checkpoint is corruption free is presented in [4], but considers a fixed recovery time that does not change based on how much state is corrupted.

\section{CONCLUSION}

As HPC systems trend toward larger numbers of smaller components at lower voltages, the rate of errors due to hardware faults is expected to increase. To mitigate these issues in HPC applications, many SDC detection and recovery schemes have been proposed. This paper explores how state corruption due to a soft error propagates at a micro (instruction level) and macro (application variable) level for three applications: Jacobi, HPCCG, and CoMD.

At a micro-level, latency of segmentation faults, divergence of control flow and detection are investigated. In addition, the impact of compiler optimizations is explored on two kernels: WAXPBY and SpMV. Results show that the majority of segmentation faults occur shortly after a fault occurs allowing for little propagation. Deviations in control flow predominantly occur as premature loop termination in loops where the fault occurs.

Macro-level results highlight the speed and intensity of corruption on the processes where the failure occurs and between other processes. Corruption results for critical data structures are discussed along with the ability to define regions of containment for local recovery. Finally, increasing the local problem size increases the latency of inter-process corruption propagation in Jacobi, while decreases the latency in CoMD (and does not influence the latency for HPCCG).

Latencies are useful in determining the effectiveness of a detection scheme - i.e., short latencies limit corruption propagation and can lower recovery costs. However, low cost recovery in a parallel application requires knowing the corrupted processes and data structures. Tracking propagation at the macro-level enables discovery of the variables that are most susceptible to corruption, it identifies the speed of corruption between processes, and indicated whether operations reduce or amplify corruption. The probability of corruption is valuable in developing more precise SDC detectors.

\section{ACKNOWLEDGMENT}

We would like to our reviewers and shepherd for their helpful and insightful comments on improving the quality of this paper.

This work was sponsored by the Air Force Office of Scientific Research under grant FA9550-12-1-0478. This work was supported in part by the Office of Advanced Scientific Computing Research, Office of Science, U.S. Department of Energy award DE-FG0213ER26138/DE-SC0010049. This material is based upon work supported by the National Science Foundation under Grant No. SHF1617488. This research is part of the Blue Waters sustained-petascale computing project, which is supported by the National Science Foundation (awards OCI-0725070 and ACI-1238993) and the state of Illinois. Blue Waters is a joint effort of the University of Illinois at Urbana-Champaign and its National Center for Supercomputing Applications.

\section{REFERENCES}

[1] E. Agullo, L. Giraud, A. Guermouche, J. Roman, and M. Zounon. Towards resilient parallel linear krylov solvers: recover-restart strategies. Rapport de recherche RR-8324, INRIA, July 2013.

[2] R. A. Ashraf, R. Gioiosa, G. Kestor, R. F. DeMara, C.-Y. Cher, and P. Bose. Understanding the propagation of transient errors in HPC applications. In Proceedings of the International Conference for High Performance Computing, Networking, Storage and Analysis, SC '15, pages 72:1-72:12, New York, NY, USA, 2015. ACM.

[3] R. C. Baumann. Radiation-induced soft errors in advanced semiconductor technologies. Device and Materials Reliability, IEEE Transactions on, 5(3):305-316, Sept. 2005.

[4] L. Bautista-Gomez, A. Benoit, A. Cavelan, S. K. Raina, Y. Robert, and H. Sun. Which verification for soft error detection? In $H i P C$, pages 2-11. IEEE Computer Society, 2015.

[5] L. Bautista-Gomez and F. Cappello. Detecting silent data corruption for extremescale MPI applications. In Proceedings of the 22Nd European MPI Users' Group Meeting, EuroMPI '15, pages 12:1-12:10, New York, NY, USA, 2015. ACM.

[6] L. Bautista-Gomez and F. Cappello. Exploiting spatial smoothness in HPC applications to detect silent data corruption. In Proceedings of the 2015 IEEE 17th International Conference on High Performance Computing and Communications, 2015 IEEE 7th International Symposium on Cyberspace Safety and Security, and 2015 IEEE 12th International Conf on Embedded Software and Systems, HPCCCSS-ICESS '15, pages 128-133, Washington, DC, USA, 2015. IEEE Computer Society.

[7] L. Bautista-Gomez, S. Tsuboi, D. Komatitsch, F. Cappello, N. Maruyama, and S. Matsuoka. FTI: high performance fault tolerance interface for hybrid systems. In Proceedings of 2011 International Conference for High Performance Computing, Networking, Storage and Analysis, SC '11, pages 32:1-32:32, New York, NY, USA, 2011. ACM.

[8] E. Berrocal, L. Bautista-Gomez, S. Di, Z. Lan, and F. Cappello. Lightweight silent data corruption detection based on runtime data analysis for HPC applications. In Proceedings of the 24th International Symposium on High-Performance Parallel 
and Distributed Computing, HPDC '15, pages 275-278, New York, NY, USA, 2015 ACM.

[9] S. Borkar. Designing reliable systems from unreliable components: The challenges of transistor variability and degradation. IEEE Micro, 25(6):10-16, Nov. 2005.

[10] P. G. Bridges, K. B. Ferreira, M. A. Heroux, and M. Hoemmen. Fault-tolerant linear solvers via selective reliability. CoRR, abs/1206.1390, 2012

[11] J. Calhoun, L. Olson, and M. Snir. FlipIt: An LLVM based fault injector for HPC. In Proceedings of the 20th International Euro-Par Conference on Parallel Processing (Euro-Par '14), 2014.

[12] J. Calhoun, L. Olson, M. Snir, and W. D. Gropp. Towards a more fault resilient multigrid solver. In Proceedings of the Symposium on High Performance Computing, HPC '15, pages 1-8, San Diego, CA, USA, 2015. Society for Computer Simulation International.

[13] J. Calhoun, M. Snir, L. Olson, and M. Garzaran. Understanding the propagation of error due to a silent data corruption in a sparse matrix vector multiply. In Proceedings of the 2015 IEEE International Conference on Cluster Computing, CLUSTER '15, pages 541-542, Washington, DC, USA, 2015. IEEE Computer Society.

[14] J. Chung, I. Lee, M. Sullivan, J. H. Ryoo, D. W. Kim, D. H. Yoon, L. Kaplan, and M. Erez. Containment domains: A scalable, efficient, and flexible resilience scheme for exascale systems. In Proceedings of the International Conference on High Performance Computing, Networking, Storage and Analysis, SC '12, pages 58:1-58:11, Los Alamitos, CA, USA, 2012. IEEE Computer Society Press.

[15] T. J. Dell. A white paper on the benefits of chipkillcorrect ECC for PC server main memory. Technical report, IBM Microelectronics Division, 1997.

[16] J. Elliott, M. Hoemmen, and F. Mueller. Evaluating the impact of SDC on the GMRES iterative solver. In Proceedings of the 2014 IEEE 28th International Paralle and Distributed Processing Symposium, IPDPS '14, pages 1193-1202, Washington, DC, USA, 2014. IEEE Computer Society.

[17] J. Elliott, F. Mueller, M. Stoyanov, and C. Webster. Quantifying the impact of single bit flips on floating point arithmetic. Technical report, Oak Ridge National Laboratory, August 2013.

[18] B. Fang, Q. Lu, K. Pattabiraman, M. Ripeanu, and S. Gurumurthi. ePVF: an enhanced program vulnerability factor methodology for cross-layer resilience analysis. 2016 46th Annual IEEE/IFIP International Conference on Dependable Systems and Networks (DSN), 00:168-179, 2016.

[19] D. Fiala, F. Mueller, C. Engelmann, R. Riesen, K. Ferreira, and R. Brightwell. Detection and correction of silent data corruption for large-scale high-performance computing. In Proceedings of the International Conference on High Performance Computing, Networking, Storage and Analysis, SC '12, pages 78:1-78:12, Los Alamitos, CA, USA, 2012. IEEE Computer Society Press.

[20] W. Gu, Z. Kalbarczyk, R. K. Iyer, and Z.-Y. Yang. Characterization of linux kernel behavior under errors. In DSN, pages 459-468. IEEE Computer Society, 2003.

[21] A. Guermouche, T. Ropars, E. Brunet, M. Snir, and F. Cappello. Uncoordinated checkpointing without domino effect for send-deterministic MPI applications. In Parallel \& Distributed Processing Symposium (IPDPS), 2011 IEEE International, pages 989-1000. IEEE, 2011.

[22] A. Guermouche, T. Ropars, M. Snir, and F. Cappello. Hydee: Failure containment without event logging for large scale send-deterministic MPI applications. In Parallel \& Distributed Processing Symposium (IPDPS), 2012 IEEE 26th International, pages 1216-1227. IEEE, 2012.

[23] S. K. S. Hari, S. V. Adve, and H. Naeimi. Low-cost program-level detectors for reducing silent data corruptions. In Proceedings of the 2012 42Nd Annual IEEE/IFIP International Conference on Dependable Systems and Networks (DSN), DSN '12, pages 1-12, Washington, DC, USA, 2012. IEEE Computer Society.

[24] A. Hassani, A. Skjellum, P. V. Bangalore, and R. Brightwell. Practical resilient cases for fa-mpi, a transactional fault-tolerant mpi. In Proceedings of the $3 \mathrm{rd}$ Workshop on Exascale MPI, ExaMPI '15, pages 1:1-1:10, New York, NY, USA, 2015 ACM.

[25] L. Jaulmes, M. Casas, M. Moretó, E. Ayguadé, J. Labarta, and M. Valero. Exploiting asynchrony from exact forward recovery for DUE in iterative solvers. In Proceedings of the International Conference for High Performance Computing, Networking, Storage and Analysis, SC '15, pages 53:1-53:12, New York, NY, USA, 2015. ACM.

[26] H. Kaul, M. Anders, S. Hsu, A. Agarwal, R. Krishnamurthy, and S. Borkar. Nearthreshold voltage (NTV) design: Opportunities and challenges. In Proceedings of the 49th Annual Design Automation Conference, DAC '12, pages 1153-1158, New York, NY, USA, 2012. ACM.

[27] D. S. Khudia, G. Wright, and S. Mahlke. Efficient soft error protection for commodity embedded microprocessors using profile information. In Proceedings of the 13th ACM SIGPLAN/SIGBED International Conference on Languages, Compilers, Tools and Theory for Embedded Systems, LCTES '12, pages 99-108, New York, NY, USA, 2012. ACM.

[28] I. Laguna, M. Schulz, D. F. Richards, J. Calhoun, and L. Olson. IPAS: intelligent protection against silent output corruption in scientific applications. In Proceed ings of the 2016 International Symposium on Code Generation and Optimization, CGO 2016, pages 227-238, New York, NY, USA, 2016. ACM.

[29] C. Lattner and V. Adve. LLVM: a compilation framework for lifelong program analysis \& transformation. In Proceedings of the 2004 International Symposium on
Code Generation and Optimization (CGO'04), Palo Alto, California, Mar 2004.

[30] G. Li, Q. Lu, and K. Pattabiraman. Fine-grained characterization of faults causing long latency crashes in programs. In DSN, pages 450-461. IEEE Computer Society, 2015.

[31] G. Li, K. Pattabiraman, C.-Y. Cher, and P. Bose. Understanding error propagation in GPGPU applications. In Proceedings of the International Conference for High Performance Computing, Networking, Storage and Analysis, SC '16, pages 21:121:12, Piscataway, NJ, USA, 2016. IEEE Press.

[32] Q. Lu, K. Pattabiraman, M. S. Gupta, and J. A. Rivers. SDCTune: a model for predicting the sdc proneness of an application for configurable protection. In Proceedings of the 2014 International Conference on Compilers, Architecture and Synthesis for Embedded Systems, CASES '14, pages 23:1-23:10, New York, NY, USA, 2014. ACM.

[33] R. E. Lyons and W. Vanderkulk. The use of triple-modular redundancy to improve computer reliability. IBM 7. Res. Dev., 6(2):200-209, Apr. 1962.

[34] T. C. May and M. H. Woods. Alpha-particle-induced soft errors in dynamic memories. Electron Devices, IEEE Transactions on, 26(1):2-9, Jan. 1979.

[35] A. Moody, G. Bronevetsky, K. Mohror, and B. R. d. Supinski. Design, modeling, and evaluation of a scalable multi-level checkpointing system. In Proceedings of the 2010 ACM/IEEE International Conference for High Performance Computing, Networking, Storage and Analysis, SC '10, pages 1-11, Washington, DC, USA, 2010. IEEE Computer Society.

[36] X. Ni and L. V. Kale. FlipBack: automatic targeted protection against silent data corruption. In Proceedings of the International Conference for High Performance Computing, Networking, Storage and Analysis, SC '16, pages 29:1-29:12, Piscataway, NJ, USA, 2016. IEEE Press.

[37] X. Ni, E. Meneses, N. Jain, and L. V. Kale. ACR: automatic checkpoint/restart for soft and hard error protection. In ACM/IEEE International Conference for High Performance Computing, Networking, Storage and Analysis, SC '13. IEEE Computer Society, Nov. 2013.

[38] K. Pattabiraman, R. K. Iyer, and Z. T. Kalbarczyk. Automated derivation of application-aware error detectors using static analysis: The trusted illiac approach. IEEE Transactions on Dependable and Secure Computing, 8:44-57, 2009.

[39] K. Pattabiraman, N. M. Nakka, Z. T. Kalbarczyk, and R. K. Iyer. SymPLFIED: symbolic program-level fault injection and error detection framework. IEEE Transactions on Computers, 62(11):2292-2307, 2013.

[40] N. Sasaki, K. Sato, T. Endo, and S. Matsuoka. Exploration of lossy compression for application-level checkpoint/restart. In Proceedings of the 2015 IEEE International Parallel and Distributed Processing Symposium, IPDPS '15, pages 914-922, Washington, DC, USA, 2015. IEEE Computer Society.

[41] S. K. Sastry Hari, R. Venkatagiri, S. V. Adve, and H. Naeimi. GangES: gang error simulation for hardware resiliency evaluation. In Proceeding of the 41st Annual International Symposium on Computer Architecuture, ISCA '14, pages 61-72, Piscataway, NJ, USA, 2014. IEEE Press.

[42] M. Snir, R. W. Wisniewski, J. A. Abraham, S. V. Adve, S. Bagchi, P. Balaji, J. Belak, P. Bose, F. Cappello, B. Carlson, A. A. Chien, P. Coteus, N. A. DeBardeleben, P. C. Diniz, C. Engelmann, M. Erez, S. Fazzari, A. Geist, R. Gupta, F. Johnson, S. Krishnamoorthy, S. Leyffer, D. Liberty, S. Mitra, T. Munson, R. Schreiber, J. Stearley, and E. V. Hensbergen. Addressing failures in exascale computing. International fournal of High Performance Computing Applications, 28(2):127 171, May 2014

[43] V. Sridharan, N. DeBardeleben, S. Blanchard, K. B. Ferreira, J. Stearley, J. Shalf, and S. Gurumurthi. Memory errors in modern systems: The good, the bad, and the ugly. In Proceedings of the Twentieth International Conference on Architectural Support for Programming Languages and Operating Systems, ASPLOS '15, pages 297-310, New York, NY, USA, 2015. ACM.

[44] N. Wang, M. Fertig, and S. Patel. Y-branches: When you come to a fork in the road, take it. In Proceedings of the 12th International Conference on Parallel Architectures and Compilation Techniques, PACT '03, pages 56-, Washington, DC, USA, 2003. IEEE Computer Society.

[45] K. S. Yim, Z. T. Kalbarczyk, and R. K. Iyer. Quantitative analysis of long-latency failures in system software. In Proceedings of the 2009 15th IEEE Pacific Rim International Symposium on Dependable Computing, PRDC '09, pages 23-30, Washington, DC, USA, 2009. IEEE Computer Society.

[46] J. Yu, M. J. Garzaran, and M. Snir. ESoftCheck: removal of non-vital checks for fault tolerance. In Proceedings of the 7th Annual IEEE/ACM International Symposium on Code Generation and Optimization, CGO '09, pages 35-46, Washington, DC, USA, 2009. IEEE Computer Society.

[47] G. Zheng, L. Shi, and L. V. Kalé. FTC-Charm++: an in-memory checkpoint-based fault tolerant runtime for Charm++ and MPI. In Cluster Computing, 2004 IEEE International Conference on, pages 93-103. IEEE, 2004. 